Article

\title{
Impact of the COVID-19 Lockdown on Lifestyle Behaviors and their association with personality among adults in Qatar: A Cross-Sectional Study
}

\author{
Tamara Al-Abdi ${ }^{1}$, Alexandros Heraclides ${ }^{2}$, Alexia Papageorgiou ${ }^{3}$ and Elena Philippou ${ }^{4,5 *}$
}

1 Human Nutrition Department, Qatar University, Qatar; tamara.alabdi@qu.edu.qa

2 Department of Heatlh Sciences, School of Sciences, European University Cyprus, Cyprus;

A.Heraclides@euc.ac.cy

3 Department of Primary Care and Population Health, University of Nicosia, Cyprus; Papageorgiou.a@unic.ac.cy

4 Department of Life and Health Sciences, School of Sciences and Engineering, University of Nicosia, Cyprus;

5 Department of Nutritional Sciences, King's College London, London, U.K; Philippou.e@unic.ac.cy

* Correspondence: Philippou.e@unic.ac.cy; Tel.: +357 99611272

\begin{abstract}
The coronavirus pandemic (COVID-19) resulted in lockdowns and social distancing measures enforced by governments. Using a cross-sectional design, this study aimed to identify changes in adherence to the Mediterranean diet (MD) and physical activity (PA) and associations with personality, during lockdown in Qatar. A sample of 543 participants was recruited online between April and May 2020. Results showed a reduction in Mediterranean diet (MD) adherence during lockdown $(5.9 \pm 0.08)$ compared to before lockdown $(6.1 \pm 0.08)(p<0.001)$. Although there was an increase in the percentage of participants who consumed $\geq 4$ tablespoons olive oil per day ( $9 \%$ vs $12 \%$; $<<0.001)$, vegetables $(54.3 \%$ vs $58.7 \%$; $=0.005)$, legumes $(11.8 \%$ vs $15.3 \%$; $p=0.007)$ and sofrito $(70.9 \%$ vs $77.3 \%$; $<0.001)$, there was also a significant decrease in the percentage of participants who consumed fresh fruit $(39.4 \%$ vs $15.8 \%$; p < 0.001$)$ and fish/seafood (5.9\% vs 3.9\%; $\mathrm{p}=0.035)$ and an increase in saturated fat consumption $(45.9 \%$ vs $53.8 \%$; $<0.001)$, during lockdown compared to before lockdown. Participants who scored high in the extraversion personality dimension had a higher $\mathrm{MD}$ adherence $(\mathrm{B}=0.84 ; 95 \% \mathrm{CI}=0.04,1.64 ; \mathrm{p}=0.039)$ before lockdown compared to participants who scored high in the agreeableness personality dimension, although this difference did not reach statistical significance during lockdown. Those who scored high in openness had the lowest change in MD adherence score $(B=-0.31 ; 95 \% \mathrm{CI}=-0.58,-0.04 ; \mathrm{p}$ $=0.026)$. Total PA $(\mathrm{B}=-506.26 ; 95 \% \mathrm{CI}=-678.60,-333.92 ; \mathrm{p}<0.001)$, vigorous activity $(\mathrm{B}=-155.95$; $95 \% \mathrm{CI}=-274.64,-38.21 ; \mathrm{p}=0.010)$, moderate activity $(\mathrm{B}=-93.04 ; 95 \% \mathrm{CI}=-148.07,-38.01 ; \mathrm{p}=$ $0.010)$ and walking $(\mathrm{B}=-257.27 ; 95 \% \mathrm{CI}=-337.87,-176.67 ; \mathrm{p}<0.001)$ were decreased during lockdown, while sitting was increased compared to before lockdown $(B=940.91,95 \% \mathrm{CI}=831.9$, 1049.90; $\mathrm{p}<0.001)$. Openness was positively associated with all PA $(B=562.2 ; 95 \% \mathrm{CI}=62.7$, 106.7; $p=0.027)$, including walking $(B=241.7 ; 95 \% \mathrm{CI}=29.4,454.0 ; p=0.026)$ and negatively associated with sitting $(B=-303.4 ; 95 \% \mathrm{CI}=-590.0 ;-16.8 ; p=0.038)$ when compared to those with high agreeableness scores, before lockdown. During lockdown, the time spent sitting was lower in those with high scores on neuroticism when compared to those with high agreeableness scores $(B=-619.5 ; 95 \% C I=-1215,-23.9 ; p=0.042)$. Differential changes in lifestyle habits by personality dimensions during lockdown, suggest the need to tailor lifestyle interventions based on people's personality types, for more effective lifestyle change.
\end{abstract}

Keywords: COVID-19, lockdown, Mediterranean diet, personality traits, physical activity, lifestyle habits 


\section{Introduction}

The coronavirus disease 2019 (COVID-19), was first reported in Wuhan, China, in December 2019, and has since massively spread all over the globe [1]. By March 11 th 2020, the World Health Organization (WHO) declared the outbreak a pandemic [2]. In order to contain the spread of the virus, countries adopted protective measures to avoid infection, which included social distancing, home confinement and closure of businesses and schools. In Qatar, such lockdown measures were declared beginning of March 9th 2020 [3]. The Qatari population was required to stay at home and only go out for essential needs, while only essential workers were allowed to work with physical distancing guidelines. On June $15^{\text {th }} 2020$, Qatar lifted lockdown restrictions and slowly reopened in 4 phases to include parks, shops and restaurants [4].

While these strict preventive measures were considered necessary, they may have changed lifestyle-related daily habits. Home confinement can affect diet, food choice and lifestyle patterns $[5,6]$. It can lead to overconsumption or even restriction of intake due to inactivity [7]. Nutritional habits could also change due to the lack of availability and limited access to food [8]. Purchasing packaged and long-life food rather than fresh food could discourage consumers from consuming the recommended portions of fruits and vegetables [9]. The changes in routine can worsen eating habits and sedentary lifestyle which can have detrimental effects on health. A recent study of 37,252 French adults found that COVID-19 lockdown resulted in consumption of unhealthy foods and an increase in body weight [10]. In regards to nutritional behaviors, $56.2 \%$ of participants reported modifying their eating habits with increased snacking, decreased consumption of fresh food products (mainly fruit and fish) and increased consumption of sweets, biscuits and cakes [10]. Another online study by Ammar et al [11] of 1047 participants, from Europe, North Africa, Western Asia and the Americas, found that COVID-19 home lockdown had a negative effect on unhealthy patterns of food consumption such as the type of food consumed, eating out of control, consuming snacks between meals, and the number of main meals. These food-related changes in behavior could be attributed to anxiety and boredom evoked by quarantine conditions. Boredom, for example has been associated with a greater energy intake [12]. Negative emotions could also lead to emotional eating especially of comfort foods which tend to be high in salt, fats and sugars [13]. A study in Italy of 1929 participants, reported an increase in "comfort food" consumption, especially desserts, chocolate, and ice-cream with $46.1 \%$ of the participants reporting eating more during quarantine and 19.5\% reporting weight gain [14]. Similar findings were reported in studies within the Gulf region. Mughamis et al [15] found a high prevalence of unhealthy eating habits and sedentary lifestyle during the pandemic, which contributed to a significant weight increase among the Kuwaiti population. This cross sectional study of 522 participants, mainly female, also reported an increase in frequency of snacks, binge eating and night-time consumption [15].

Not all studies found a negative association between the COVID-19 pandemic and dietary habits. A study, using an online questionnaire, on 579 respondents living in Qatar, reported a shift towards a healthier diet during the COVID-19 pandemic [3]. Consumers reduced their consumption of unhealthy foods such as fast food, unhealthy snacks, candy, cookies, cakes and pastries. They reported eating healthier, including more fresh fruit and vegetables and also consuming healthy snacks [3].

The COVID-19 lockdown measures have also resulted in changes in physical activity. The closure of gyms and enforcement of curfews in several countries has resulted in limiting participation in both indoor and outdoor activities. Restrictions have reduced overall physical activity, time, frequency and access to exercise. Several studies have reported a decrease in all levels of physical activity, vigorous, moderate and walking, during COVID-19 lockdown and an increase in sedentary behaviors [5,11,16]. A study of 
1844 participants in Jordan, reported a $42.4 \%$ decrease in activities such as walking, jogging and sports during COVID-19 lockdown, while $82.7 \%$ participants reported increase in sedentary behaviors such as watching TV and using electronics [17].

Personality traits are among the variables predicting types of action that people employ. Existing studies have attested to the association between personality traits and health behavior [18]. Of the personality traits defined by the five-factor model (FFM), conscientiousness and neuroticism emerge as the most relevant predictors of health behaviors [19-22]. As recently reviewed [23], individuals who score high in conscientiousness tend to consume breakfast, have their meals at regular times each day and exercise more. On the other hand, neuroticism was positively correlated with emotional eating and consumption of sweet foods, eating less fruit and vegetables and physical inactivity [23].

Although it is known that personality traits are stable and tend to be resistant to stressful life events [24, 25], individual differences in personality may contribute to how people respond and behave during a pandemic. Personality may shape such responses through beliefs and attitudes associated with the different traits [26].

Limited studies have examined the association between personality traits and health behaviors during the COVID-19 pandemic. A recent study by Kroencke et al [27] found that individuals scoring high in neuroticism experienced a more negative effect in their daily lives during the pandemic. Another study [28] found that the pandemic did not influence the wellbeing of those who scored high in openness and conscientiousness.

The rigorous restrictions set in Qatar, may have affected dietary intake and exercise habits. Nevertheless, there is limited evidence to evaluate the effect on confinement on these lifestyle factors and no known evidence linking personality to these factors. The main aim of this study was thus to investigate for the first time the immediate impact of COVID-19 lockdown on diet and exercise and the associations with personality and sociodemographics.

\section{Materials and Methods}

\subsection{Study Design and Participants}

A cross sectional study was conducted from $24^{\text {th }}$ April to $23^{\text {rd }}$ May 2020, during which time Qatar was under COVID-19 lockdown which included social distancing, home confinement and closure of businesses and schools. The Qatari population were required to stay at home and only go out for essential needs (food or medicine). For those who did leave the house, only one individual was allowed to be in the car when going out to purchase either food or medicine. With regards to work, only essential workers, such as health care workers, were allowed out with physical distancing guidelines. The target population was residents of Qatar, aged 18 years or older recruited through social media (WhatsApp and Twitter) and by email using a snowball convenience sampling method [29]. Respondents were asked to complete anonymously an online questionnaire that was distributed through social media and also encouraged to distribute the questionnaire to their contacts residing in Qatar. Participants were asked to provide written informed consent prior to completing the questionnaire. Ethical approval for this study was obtained from Qatar University Institutional Review Board (QU-IRB 1081-EA/19) and it was conducted in accordance with the Declaration of Helsinki.

\subsection{Questionnaire}

The questionnaire consisted of 95 questions structured into 5 sections: (1) Socio demographics, (2) Dietary Habits before and during COVID-19 lockdown, (3) Physical activity before and during COVID-19 lockdown, (4) Personality and (5) Chrononutrition as described in detail below. 


\subsubsection{Socio Demographic Data}

Participants were asked to report their age, gender, nationality, highest education attained (middle school, high school, university undergraduate, university postgraduate), marital status (single, married, divorced, separated, widowed), employment status (student, employed, unemployed, retired), smoking, weight and height during the lockdown.

\subsubsection{Dietary Assessment}

Dietary habits were assessed using the MD Adherence Screener (MEDAS), which has been shown to be a valid tool for assessment of MD adherence [30]. The questionnaire comprises of 14 questions which assess intake of: olive oil as main fat, tablespoons of olive oil used, vegetables, fruit and fruit juices, meat and meat products, fats (butter, margarine, cream), sweet or carbonated beverages, wine, legumes, fish or shellfish, commercial sweets or pastries, unsalted nuts, poultry and sofrito sauce. Taking into consideration cultural and religious practices in Qatar, the component related to alcohol (i.e. wine) consumption was not included in the present questionnaire, leaving only 13 components of the MEDAS score and thus the score range of 0-13. Additionally, 'ghee' was added under the question regarding fat consumption since this is commonly used in Qatar.

\subsubsection{Physical Activity Assessment}

The short version of the International Physical Activity Questionnaire (IPAQ-SF) was used to assess physical activity [31]. IPAQ is a validated tool extensively used to categorize individuals as having low, medium or high PA levels based on the frequency and duration of activities of various intensities and the time spent seated over the past 7 days [32]. The weekly PA level was calculated as energy expenditure in Metabolic Equivalent Task-minutes/week (MET-min/wk), using the assigned MET for each task (3.3 for walking, 4.0 for moderate-intensity PA, and 8.0 for vigorous-intensity PA). Computations of MET-mins/wk were calculated following the IPAQ recommendations for scoring protocol [31].

\subsubsection{Personality}

Personality traits were assessed using the 44-item validated short version of the Big Five Inventory (BFI-10) [33, 34] which classifies these traits into five broad dimensions: agreeableness, extraversion, conscientiousness, neuroticism and openness.

\subsubsection{Chrononutrition}

The Chrononutrition Profile - Questionnaire (CP-Q) was used to assess chrononutrition behaviors, which includes first and last eating events, preferred morning and evening latency, and preferred eating window [35]. Results on this part of the questionnaire will be analysed and reported in another paper.

\subsection{Statistical Analysis}

Socio-demographic variables were re-categorized before analysis. Respondent's age was re-categorized into 4 categories ( $\leq 20$ years; $21-29$ years; $30-39$ years; $\geq 40$ years). Nationality was re-categorized into 2 categories (Arab and non-Arab). Education was recategorized into 3 categories (secondary; undergraduate University; postgraduate University). Self-reported data on height and weight were used to calculate BMI $\left(\mathrm{kg} / \mathrm{m}^{2}\right)$ and interpreted according to WHO classification as underweight (BMI: $18.5 \mathrm{~kg} / \mathrm{m}^{2}$ ), normal weight (BMI: 18.5-25.0 kg/m²), overweight (BMI: 25.0-30.0 kg/m²) and obese (BMI: $30 \mathrm{~kg} / \mathrm{m}^{2}$ ). Distribution of numeric variables was assessed using normality plots. Paired $\mathrm{t}$ 
tests were used to determine the association between paired numeric variables, the McNemar test for paired binary variables and the Bowker's Symmetry test for categorical variables with $>2$ categories (e.g. MD adherence: low, moderate, high), before and during the COVID-19 lockdown. Association of personality dimensions with dietary habits (MD adherence) and PA were investigated in multiple linear regression models using personality as the main independent variable of interest. No adjustments were made in the first regression model, while the second model was adjusted for gender, age, nationality, education, marital status, employment, smoking and BMI. Data are presented as number and percentage in parentheses (\%) for categorical variables and mean +/standard deviation for continuous variables. Results were considered statistically significant if the $\mathrm{p}$-value was $<0.05$. Data analysis was done using STATA (Version 16, Stata Corporation, and College Station, TX, USA).

\section{Results}

\subsection{Participants' sociodemographic characteristics}

A total of 560 participants completed the questionnaire, of which 17 were excluded due to incomplete data, leaving a final sample of 543 participants. The demographic characteristics of the participants are shown in Table 1. In brief, most of the participants were female $(88.4 \%, \mathrm{n}=480)$ and almost half $(44.8 \%, \mathrm{n}=243)$ were aged between $21-29$ years. Most were Arab $(73.7 \%, \mathrm{n}=400)$, students $(46 \%, \mathrm{n}=250)$, with an undergraduate level of education $(77.7 \%, \mathrm{n}=422)$, single $(63.4 \%, \mathrm{n}=344)$ and nonsmokers $(91.9 \%, \mathrm{n}=$ 499). Regarding body weight, $31.3 \%(n=170)$ were classified as overweight (BMI = 25.0$\left.29.9 \mathrm{~kg} / \mathrm{m}^{2}\right)$ and $13.3 \%(\mathrm{n}=72)$ were classified as obese $\left(\mathrm{BMI}=\geq 30 \mathrm{~kg} / \mathrm{m}^{2}\right)$. With respect to personality, the majority of participants scored high in openness $(41.4 \%, n=225)$, followed by agreeableness $(33 \%, n=179)$. Associations were found between gender as well as age and personality. In particular, males were overrepresented among those scoring high in conscientiousness $(28.6 \%, \mathrm{n}=30)$ and females were overrepresented among those scoring high in agreeableness $(95.5 \%, \mathrm{n}=171)(p<0.001)$. With regards to age, those with higher scores in openness were between the ages of 21-29 years and 30-39 years old. High scores in agreeableness were found in those aged $\leq 20$ years of age. Those who were middleaged ( $\geq 40$ years of age) had higher scores in conscientiousness $(p<0.001)$. Arab nationality was associated with high scores in openness $(p=0.020)$. Secondary, undergraduate and postgraduate level of education was also associated with high scores in openness ( $p=$ 0.004). In the category of marital status, openness was associated with being both single and married $(p<0.001)$. With respect to employment status, being a student was associated with agreeableness, being employed was associated with openness while conscientiousness was particularly frequent among those who were unemployed $(p<$ 0.001). With regards to body weight, those with high scores in agreeableness were more likely to be under or normal weight, while those with high scores in openness were more likely to be overweight and obese $(p<0.001)$.

Table 1. Characteristics of the Studied Population by Personality during the COVID-19 Lockdown 


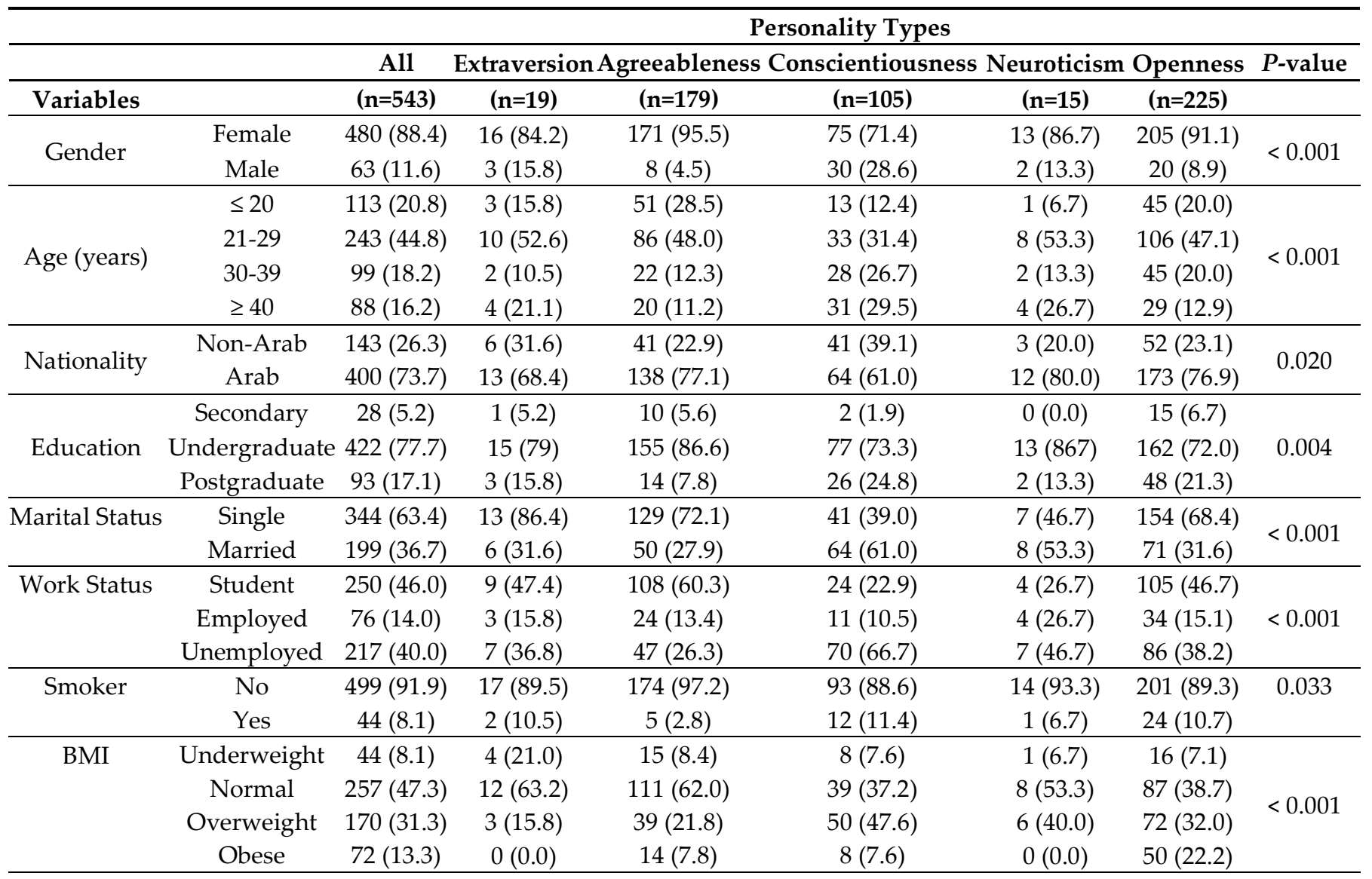

Statistical significance at the 5\% significance level using Chi-squared test. $\mathrm{n}=$ frequencies ; $\%=$ percentages.

\subsection{Adherence to the Mediterranean Diet before and during Covid-19 lockdown.}

Figure 1(A) presents the results of the adherence to the MD before and during the COVID-19 lockdown. There was a statistically significant reduction in MEDAS mean score during the studied period (before vs during lockdown: $6.1 \pm 0.08$ vs $5.9 \pm 0.08$ ) with a mean difference of $-0.20(95 \% \mathrm{CI}=-0.31,-0.90 ; P<0.001)$. More than $50 \%$ of the sample was classified as having moderate adherence to the MD both before and during lockdown as reported in Figure 1(B). A decrease was observed in those classified as having high adherence ( $11.7 \%$ vs $7.4 \%)$, whereas an increase was seen in the participants with low adherence (low adherence before vs during lockdown: $36.3 \%$ vs $41.6 \%$, p < 0.001). Of the 543 individuals, the largest contribution to this statistically significant association was a change of $2.2 \%$ of the sample from moderate adherence to high adherence and $6.3 \%$ of the sample from high adherence to moderate adherence.

The breakdown of the MEDAS components before and during COVID-19 lockdown in Qatar are presented in Figure 1(C). A statistically significant increase was seen in the percentage of participants who consumed $\geq 4$ tablespoons olive oil per day (9\% vs $12 \%$; $p$ $<0.001) ; \geq 2$ servings of raw or cooked vegetables per day $(54.3 \%$ vs $58.7 \% ; p=0.005) ; \geq 3$ 
servings of legumes per week $(11.8 \%$ vs $15.3 \% ; p=0.007)$ and $\geq 2$ servings of 'sofrito' per week $(70.9 \%$ vs $77.3 \%$; $<0.001)$ when comparing before and during lockdown. On the other hand, the percentage of participants who consumed $\geq 3$ servings of fresh fruit or fruit juice per day $(39.4 \%$ vs $15.8 \% ; p<0.001)$ and $\geq 3$ servings of fish or seafood per week (5.9\% vs 3.9\%; $p=0.035)$ significantly decreased during the lockdown compared to before the lockdown. Additionally, there was a significant increase in the percentage of participants who consumed $<1$ serving of fats (such as butter, margarine, ghee or cream) per day, when comparing before with during the lockdown $(45.9 \%$ vs $53.8 \%$; $p<0.001)$.

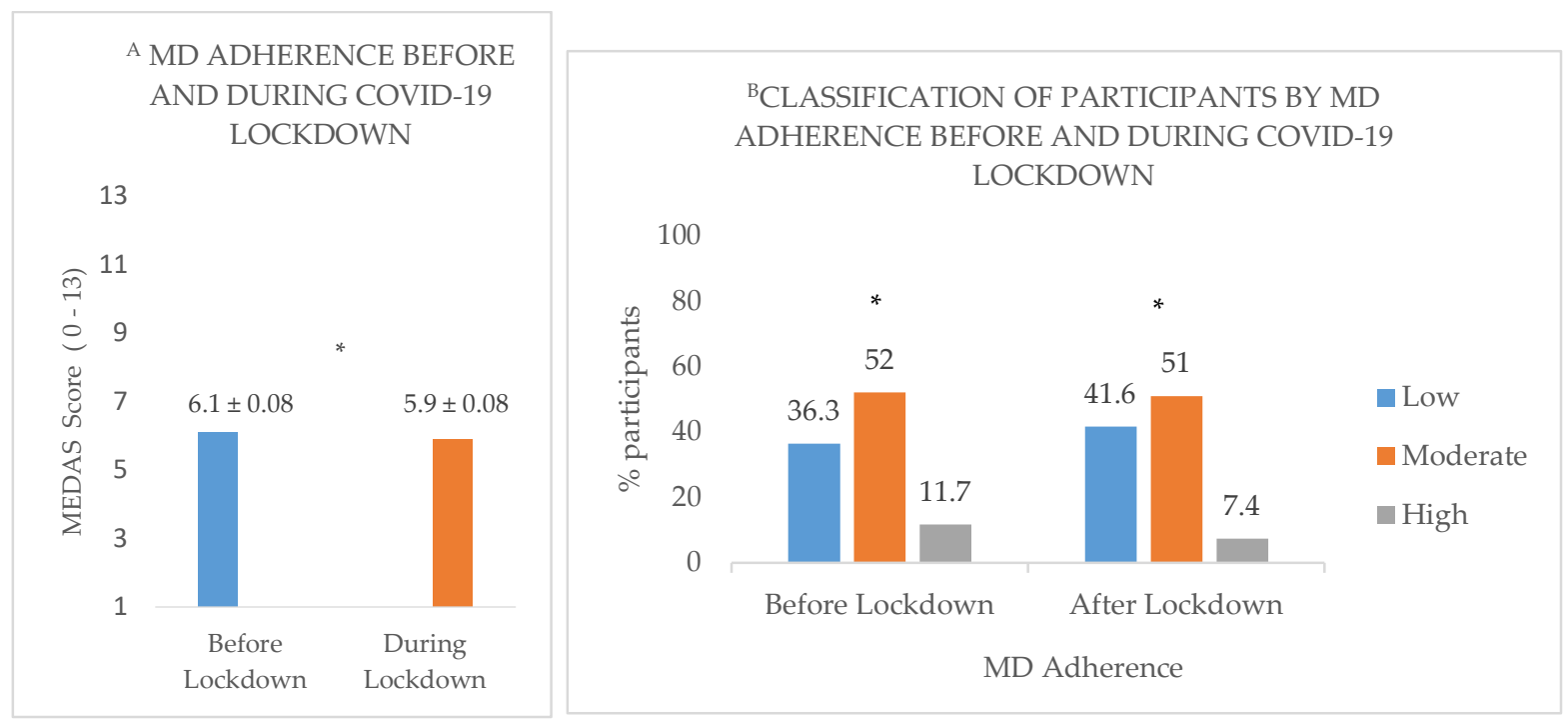




\section{C \% OF PARTICIPANTS WITH POSITIVE RESPONSES TO MEDAS QUESTIONS BEFORE AND DURING COVID-19 LOCKDOWN}

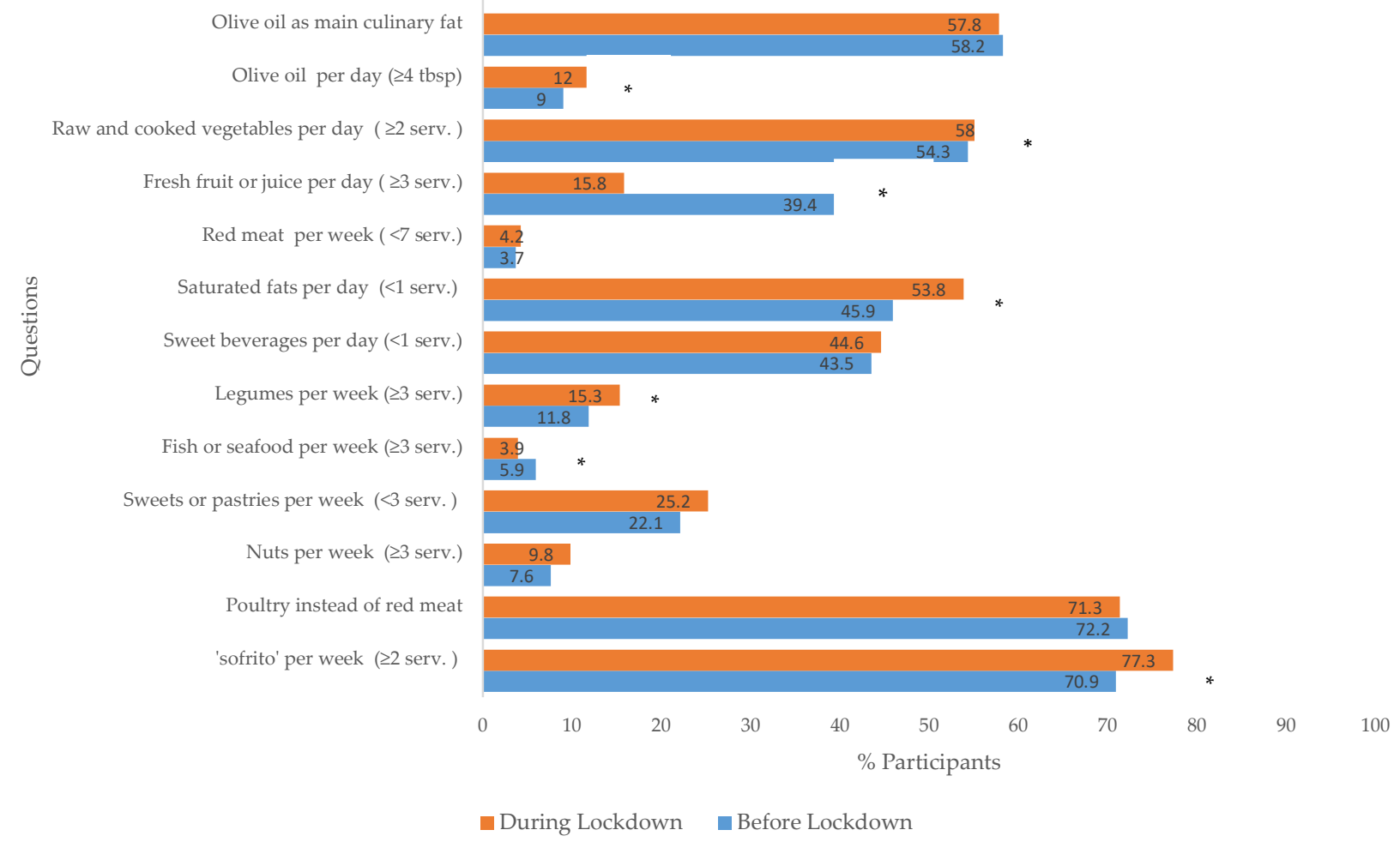

Figure 1. Dietary Habits Before and During COVID-19 Lockdown (A) MD Adherence score before and during lockdown. * indicates statistical significance at the $5 \%$ significance level using paired $t$ tests. (B) Classification of participants by MD adherence (Low $\leq 5$; Medium 6-8; High $\geq 9$ ) before and during lockdown. ${ }^{*}$ indicates statistical significance at the $5 \%$ significance level using paired $t$ test $(\mathbf{C})$ Percentage of participants with positive responses to MEDAS questions before and during COVID-19 Lockdown. Values expressed as mean \pm standard deviation. ${ }^{*}$ indicates statistical significance at the $5 \%$ significance level using Stuart-Maxwell test.

\subsection{Personalilty and Mediterranean Diet adherence before and during lockdown}

An association between the different personality dimensions and MEDAS score was found in our study population (Table 2).

Table 2. Association between MD Adherence and Types of Personality ( $n=543)$ Before and During COVID-19 Lockdown 


\section{Personality Type}

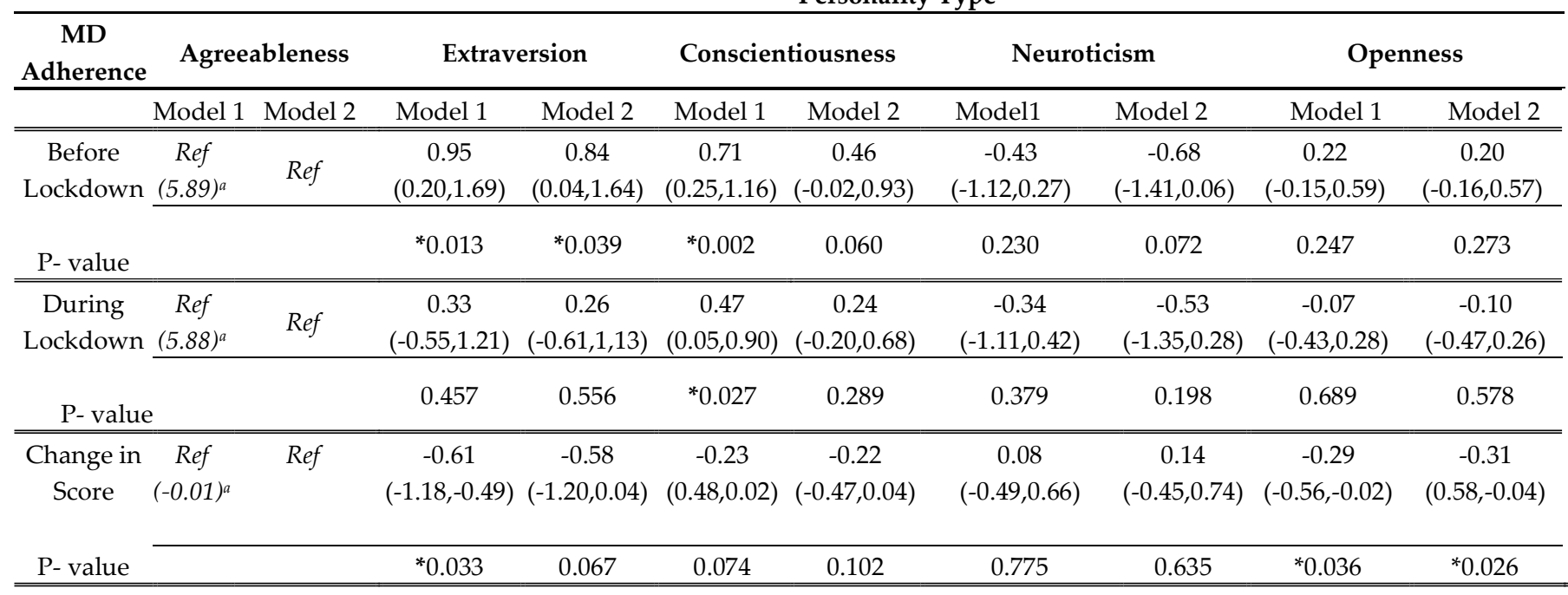

Model 1: Unadjusted

Model 2: Adjusted for gender, age, nationality, education, marital status, work status, BMI, smoking

Mean difference (95\% CI) between each personality type and the personality type agreeableness (reference category)

* indicate statistical significance at the $5 \%$ significance level using linear regression.

a indicates mean of reference category (agreeableness)

People scoring high in extraversion $(\mathrm{B}=0.95 ; 95 \% \mathrm{CI}=0.20,1.69 ; p=0.013)$ and conscientiousness $(\mathrm{B}=0.71 ; 95 \% \mathrm{CI}=0.25,1.16 ; p=0.002)$ had higher MEDAS scores compared to people scoring high in agreeableness (treated as the reference personality trait), before COVID-19 lockdown. After adjusting for confounders, the association remained robust for extraversion ( $\mathrm{B}=0.84 ; 95 \% \mathrm{CI}=0.04,1.64 ; p=0.039$ ), whereas the association with conscientiousness decreased and became marginally non-significant $(\mathrm{p}=$ 0.060). During COVID-19 lockdown, those with higher scores on conscientiousness had higher MEDAS scores compared to agreeableness $(\mathrm{B}=0.47 ; 95 \% \mathrm{CI}=0.05,0.90 ; p=0.027$ ). This association, however, was attenuated after adjusting for confounders in model 2. Concerning change in MEDAS score, both extraversion $(\mathrm{B}=-0.61 ; 95 \% \mathrm{CI}=-1.18,-0.49 ; p=$ $0.033)$ and openness $(B=-0.29 ; 95 \% C I=-0.56,-0.02 ; p=0.036)$ scores did not change as much compared to agreeableness before and during lockdown. After adjusting for confounders however, the association remained robust only for those with high openness scores $(\mathrm{B}=-0.31 ; 95 \% \mathrm{CI}=-0.58,-0.04 ; p=0.026)$. The changes in MD scores for each category can be seen in supplementary Table S1.

\subsection{Physical activity before and during Covid-19 lockdown}


Responses to the physical activity questionnaire completed before and during COVID-19 lockdown are presented in Figure 2. The total physical activity was significantly reduced from $1836.7 \pm 2087.9 \mathrm{Met} / \mathrm{min}$-week before COVID-19 lockdown compared to $1330.5 \pm 1663.7$ during COVID-19 lockdown $(B=-506.26 ; 95 \% \mathrm{CI}=-678.60$, 333.92; $p<0.001)$. Specifically, MET/min-week values of Vigorous intensity activity (867.4 \pm 1412.4 vs $711.5 \pm 1190.4 ; B=-155.95 ; 95 \% C I=-274.64,-38.21 ; p=0.010)$; Moderate intensity activity $(301.3 \pm 677.0$ vs $208.3 \pm 498.4 ; B=-93.04 ; 95 \% \mathrm{CI}=-148.07,-38.01 ; p=$ $0.010)$ and walking $(668.0 \pm 838.4$ vs $410.7 \pm 630.8 ; B=-257.27 ; 95 \% \mathrm{CI}=-337.87,-176.67 ; p$ $<0.001$ ) were significantly lower during lockdown compared to before lockdown. Sitting, on the other hand, was significantly increased during COVID-19 lockdown, with mean $\mathrm{MET} / \mathrm{min}$-week values of $2896.4 \pm 199.7$ compared to $3837.3 \pm 1181.2$ before lockdown $(B$ $=940.91,95 \% \mathrm{CI}=831.9,1049.90 ; p<0.001)$.

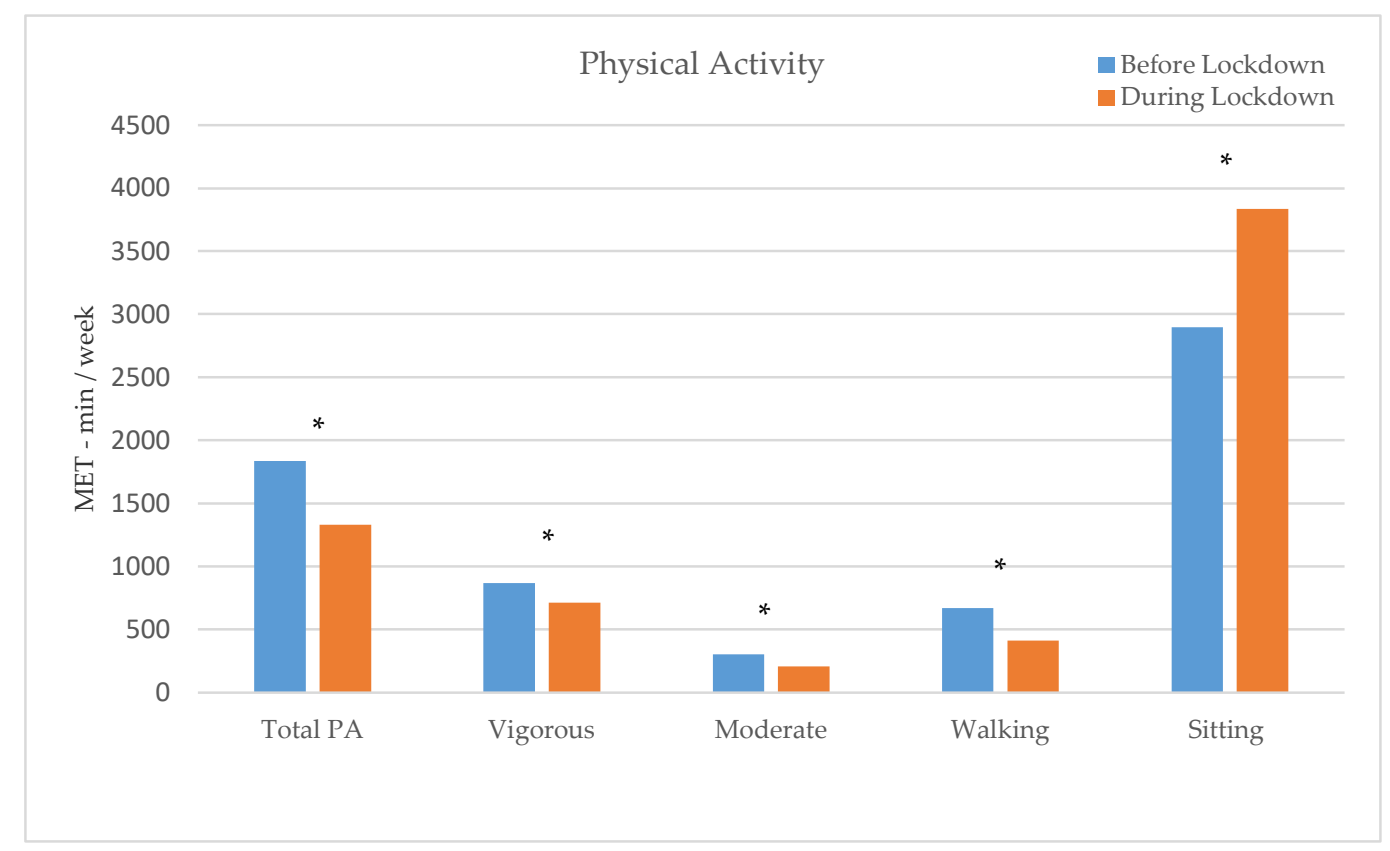

Figure 2. Comparison of PA before and during Covid-19 Lockdown. Total PA, Vigorous, Moderate PA, walking and Sitting in MET/min-week, before and during COVID-19 lockdown. Data expressed as mean values and compared by paired T tests. * indicate statistical significance at the $5 \%$ significance level.

\subsection{Personality and physical activity before and during lockdown}

Associations of personality dimensions and PA before and during COVID-19 lockdown are presented in Table 3. Among the personality dimensions examined, and using agreeableness personality as the reference, those who scored high in openness were more likely to engage in all PA $(B=562.2 ; 95 \% \mathrm{CI}=62.7,106.7 ; p=0.027)$, including walking $(B=241.7 ; 95 \% C I=29.4,454.0 ; p=0.026)$ before lockdown, even after adjusting for confounders. Those with high openness scores were also least likely to be sitting $(B=-$ 303.4; $95 \% \mathrm{CI}=-590.0 ;-16.8 ; p=0.038$ ) when compared to those with high agreeableness 
scores, before lockdown. During lockdown, this association became less apparent and did not reach statistical significance. The time spent sitting was lower in those with high scores on neuroticism when compared to those with high agreeableness scores. Although the specific comparison did not reach statistical significance before lockdown, it did during lockdown, after adjusting for confounders in model $2(B=-619.5 ; 95 \% \mathrm{CI}=-1215,-23.9 ; p=$ 0.042). Associations of other physical activity characteristics not discussed here are presented in supplementary Table S2. Additionally, the PA MET/min-week values for each personality trait are shown in supplementary Table S3. 
Table 3. Physical activity characteristics by personality traits before and during the COVID-19 Lockdown

\section{Personality Type}

PA (MET values)

Extraversion

Conscientiousness

Neuroticism

Openness

\begin{tabular}{|c|c|c|c|c|c|c|c|c|c|c|c|}
\hline & & Model 1 & Model2 & Model 1 & Model 2 & Model 1 & Model 2 & Model 1 & Model 2 & Model 1 & Model 2 \\
\hline \multirow{4}{*}{ All PA } & $\begin{array}{c}\text { Before } \\
\text { Lockdown }\end{array}$ & $\begin{array}{l}\text { Ref } \\
(1566.4)^{\mathrm{a}}\end{array}$ & Ref & $\begin{array}{c}544.9 \\
(-404.2,1514) \\
\end{array}$ & $\begin{array}{c}499.5 \\
(-513,1512) \\
\end{array}$ & $\begin{array}{c}272.8 \\
(-266.6,812.2) \\
\end{array}$ & $\begin{array}{c}221.2 \\
(-357.7,800.1) \\
\end{array}$ & $\begin{array}{c}-222.7 \\
(-1113.3,668.1) \\
\end{array}$ & $\begin{array}{c}-228.5 \\
(-137.4,680.5) \\
\end{array}$ & $\begin{array}{c}481.1 \\
(-3.9,966.2) \\
\end{array}$ & $\begin{array}{c}562.2 \\
(62.7,1061.7) \\
\end{array}$ \\
\hline & $P$-value & & & 0.256 & 0.333 & 0.321 & 0.453 & 0.623 & 0.621 & $0.052^{*}$ & $0.027^{*}$ \\
\hline & $\begin{array}{l}\text { During } \\
\text { Lockdown }\end{array}$ & $\begin{array}{l}\operatorname{Ref} \\
(1114.5)^{\mathrm{a}}\end{array}$ & Ref & $\begin{array}{c}559.5 \\
(-110.8,1230.6) \\
\end{array}$ & $\begin{array}{c}426.3 \\
(-240.3,1093) \\
\end{array}$ & $\begin{array}{c}295.7 \\
(-143.2,734.6)\end{array}$ & $\begin{array}{c}187.3 \\
(-288.2,662.7)\end{array}$ & $\begin{array}{c}193.2 \\
(-624.6,1011.2)\end{array}$ & $\begin{array}{c}102.2 \\
(-744.4,948.8)\end{array}$ & $\begin{array}{c}311.8 \\
(-78.8,702.3) \\
\end{array}$ & $\begin{array}{c}358.9 \\
(-537,771.5)\end{array}$ \\
\hline & $P$-value & & & 0.102 & 0.209 & 0.186 & 0.439 & 0.642 & 0.813 & 0.117 & 0.088 \\
\hline \multirow{4}{*}{ Walking } & $\begin{array}{c}\text { Before } \\
\text { Lockdown }\end{array}$ & $\begin{array}{l}\operatorname{Ref} \\
(584.0)^{\mathrm{a}}\end{array}$ & Ref & $\begin{array}{c}-157.8 \\
(340.8,25.2)\end{array}$ & $\begin{array}{c}-96.0 \\
(-321.3,129.3)\end{array}$ & $\begin{array}{c}34.6 \\
(-182.1,251.3) \\
\end{array}$ & $\begin{array}{c}100.0 \\
(-162.7,362.8)\end{array}$ & $\begin{array}{c}-188.0 \\
(-427.2,51.2)\end{array}$ & $\begin{array}{c}-134.2 \\
(-428.4,160.0)\end{array}$ & $\begin{array}{c}205.0 \\
(458.8,709.2) \\
\end{array}$ & $\begin{array}{c}241.7 \\
(29.4,454.0)\end{array}$ \\
\hline & $P$-value & & & 0.091 & 0.403 & 0.754 & 0.455 & 0.123 & 0.370 & $0.040^{*}$ & $0.026^{*}$ \\
\hline & $\begin{array}{c}\text { During } \\
\text { Lockdown }\end{array}$ & $\begin{array}{l}\operatorname{Ref} \\
(311.3)^{\mathrm{a}}\end{array}$ & Ref & $\begin{array}{c}139.7 \\
(-56.4,335.9) \\
\end{array}$ & $\begin{array}{c}117.3 \\
(-99.5,334.2) \\
\end{array}$ & $\begin{array}{c}146.1 \\
(-27.1,319.3) \\
\end{array}$ & $\begin{array}{c}74.5 \\
(-95.5,224.4) \\
\end{array}$ & $\begin{array}{c}14.9 \\
(-197.3,227.1) \\
\end{array}$ & $\begin{array}{c}-11.5 \\
(-229.6,206.2) \\
\end{array}$ & $\begin{array}{c}153.4 \\
(11.8,295.0) \\
\end{array}$ & $\begin{array}{c}149.2 \\
(-11.3,309.7) \\
\end{array}$ \\
\hline & $P$-value & & & 0.162 & 0.288 & 0.098 & 0.390 & 0.890 & 0.916 & $0.034^{*}$ & 0.068 \\
\hline \multirow{4}{*}{ Sitting } & $\begin{array}{c}\text { Before } \\
\text { Lockdown }\end{array}$ & $\begin{array}{c}\operatorname{Ref} \\
(3017.6)^{\mathrm{a}}\end{array}$ & Ref & $\begin{array}{c}-287.6 \\
(-949,373.7) \\
\end{array}$ & $\begin{array}{c}-322.0 \\
(-1005.2,361.2) \\
\end{array}$ & $\begin{array}{c}45.4 \\
(-310.8,407.5) \\
\end{array}$ & $\begin{array}{c}-86.0 \\
(-470.6,298.7) \\
\end{array}$ & $\begin{array}{c}-497.6 \\
(-1092.8,97.5) \\
\end{array}$ & $\begin{array}{c}-549.2 \\
(-1177.4,79.1) \\
\end{array}$ & $\begin{array}{c}-243.4 \\
(-518.4,31.7) \\
\end{array}$ & $\begin{array}{c}-303.4 \\
(-590.0,-16.8) \\
\end{array}$ \\
\hline & $P$-value & & & 0.393 & 0.355 & 0.791 & 0.661 & 0.101 & 0.086 & 0.083 & $0.038^{*}$ \\
\hline & $\begin{array}{c}\text { During } \\
\text { Lockdown }\end{array}$ & $\begin{array}{c}\operatorname{Ref} \\
(3917.6)^{\mathrm{a}}\end{array}$ & Ref & $\begin{array}{c}-347.6 \\
(-1042.5,347.2)\end{array}$ & $\begin{array}{c}-373.2 \\
(-1099.3,352.9)\end{array}$ & $\begin{array}{c}22 \\
(-325.3,369.2)\end{array}$ & $\begin{array}{c}-118.0 \\
(-499.8,263.7)\end{array}$ & $\begin{array}{c}-573.8 \\
(-1191.8,44.2)\end{array}$ & $\begin{array}{c}-619.5 \\
(-1215.2,-23.9)\end{array}$ & $\begin{array}{c}-126.3 \\
(-402.9,150.3)\end{array}$ & $\begin{array}{c}-179.5 \\
(-472.6,113.6)\end{array}$ \\
\hline & $P$-value & & & 0.326 & 0.313 & 0.901 & 0.544 & 0.069 & $0.042^{*}$ & 0.370 & 0.229 \\
\hline
\end{tabular}

Model 1: Unadjusted

Model 2: Adjusted for gender, age, nationality, education, marital status, work status, BMI, smoking

Mean difference $(95 \% \mathrm{CI}$ ) between each personality type and Agreeableness (reference category)

${ }^{*}$ indicates statistical significance at the $5 \%$ significance level using linear regression, a indicates means of reference category (agreeableness) 


\section{Discussion}

To our knowledge, this is the first study to investigate the association of personality in relation to lifestyle habits such as diet and exercise during the COVID-19 lockdown. Noticeably, there was a reduction in MD adherence during lockdown in the study population. Although lockdown was associated with an increase in olive oil, vegetables, legumes, sofrito and fat consumption, there was also a significant reduction in fresh fruit and fish/seafood consumption during lockdown. Certain personality types such as openness had higher MD adherence before but not during lockdown. Participants who scored high in openness were more likely to engage in PA (vigorous and moderate) and walking and less likely to be sitting before lockdown. Nevertheless, when examining the entire sample, total PA (vigorous, moderate) and walking were reduced during lockdown while time spent sitting was increased. During lockdown, those who scored high in neuroticism were less likely to be sitting.

Concerning demographics, women scored high in the agreeableness dimension, which was also reported in other studies [36, 37]. Our study found that the majority of men scored high in the conscientiousness dimension, which contradicts some studies that report women with higher scores in this dimension [37]. This finding however is not consistent across various cultures [37], which could explain our finding. With regards to BMI, our finding of those with high agreeableness dimension associated with low or normal BMI is consistent with previous studies [38]. Those who were overweight or obese scored high in openness dimension and this contradicts other studies which reported that those who scored high in openness were less likely to be overweight or obese [38,39]. This could also be due to cultural differences.

Overall, our study found a significant but small reduction in the MD adherence score during the COVID-19 lockdown by an average of 0.2 points. This is in contrast to other studies in Spain and Chile where participants had adopted MD eating patterns during lockdown [40,41]. As suggested by Di Renzo et al [42], the MD could be one of the best food models for strengthening immunity and protecting against COVID-19. Therefore, a reduced adherence to the MD could compromise nutritional quality, reduce intake of foods high in antioxidant and anti-inflammatory properties, and as a result increase susceptibility to viral infections. Our findings on worsening eating habits, such as a decrease in fresh fruit and fish/seafood and an increase in fat consumption, were also similarly observed in other studies [11, 43]. A study of 37,252 French adults reported that individuals bought fewer fresh products such as fruit due to limited shopping and lack of access to usual food shops leading to replacement of fruits with frozen vegetables [10]. Similarly, a study in Kuwait [44] also reported an overall significant reduction in fish and seafood consumption. The decrease in fresh fruit and seafood consumption in our findings was expected since fish and fruit 
markets in Qatar were closed during lockdown. It is also culturally preferred to consume fresh rather than frozen fish therefore contributing to reduction in consumption. On the other hand, there was improvement in some dietary components in our study during lockdown such as consumption of more olive oil, vegetables, legumes and sofrito, similar to that reported in a Spanish study [40]. These findings could be explained by the increased consumption of locally produced legumes and vegetables because of food safety concerns and uncertainty of where imported food comes from [3]. Similarly, another study looking at food behavior and consumption in Qatar, found that $32.4 \%$ of respondents increased their consumption of vegetables during lockdown [3].

With regards to PA, all types (vigorous, moderate and walking), were significantly reduced during lockdown, while sitting was increased. These results were expected considering the country was under lockdown and there was no access to gyms as well as permission to leave the house freely. Our findings are similar to other studies that reported significant reduction in PA [43] due to the limitations during lockdown [10, 44]. Another study on 1047 respondents from Asia, Africa, and Europe found that lockdown had a negative effect on all PA while daily sitting increased from 5 to 8 hours per day [11]. Our findings, however, do contradict other studies that reported an increase or maintenance of physical activity during lockdown [43]. A study on 3533 respondents aged between 12 and 86 years in Italy, reported a 9.9\% increase in training frequency in those who already took part in sports [41], while those who managed to exercise occasionally had more time to do so at home during lockdown.

With regards to personality and diet associations, our results suggest that no personality dimension was correlated with MD adherence during lockdown, after adjusting for confounders. This finding differs from other studies, which reported that those who score high in conscientiousness, are known to be organized and therefore practice dietary restraint and tend to follow a healthy dietary pattern [23]. Boggs \& Robert [45] also reported that conscientious individuals are more likely to maintain healthy diets, and exert greater levels of self-control. Nevertheless, few studies have examined health behaviors and personality traits during the COVID-19 pandemic. One study found that individuals scoring high in neuroticism experienced a greater negative impact on their daily lives during COVID-19 pandemic [27]. Another study reported that those low in extraversion and high in conscientiousness were more likely to adhere to social distancing and hygiene practices during the pandemic [46]. Although personality traits are typically stable [23,47], research has shown that traits do change in response to distress [24]. None of the studies however, have looked at personality dimensions and specific eating behaviors. A study during the COVID-19 outbreak in the US, reported changes in the five-factor model personality trait [24]. Neuroticism, for instance decreased across six weeks of lockdown, indicating that feelings of anxiety and distress associated with this personality dimension could be attributed to the pandemic itself rather than one's personality traits [24]. Furthermore, they reported acute personality changes related to isolation in those with conscientiousness, agreeableness, 
neuroticism and openness traits. Indeed, one study found that neuroticism during the COVID-19 pandemic was associated with health promoting behaviors [26]. They concluded that those who are neurotic adopt healthpromoting behaviors, unexpected during normal circumstances, due to the anxiety and fear evoked by the pandemic. Our findings similarly suggest acute changes and instability of the five factor personality traits, in relation to dietary habits, due to the COVID-19 pandemic. It is likely that those who score high in the neuroticism dimension, increase their health promoting behaviors out of worry and perceived fear of COVID-19 pandemic. Our study also found that those who scored high in openness had a decrease in MEDAS score during lockdown. This finding is supported by a literature review that also reported openness to be associated with less compliance to healthy eating recommendations [47].

In relation to personality and its association with PA, openness was positively associated with total PA before lockdown, while negatively associated with sitting among all personality dimensions. However, during lockdown there was no increase in total PA. Interestingly, though, those who scored high in neuroticism spent less time sitting compared to other personality dimensions even after adjusting for confounders. A few studies do support that those who score high in neuroticism tend to be physically active [48], tend to avoid risks and are compliant with policies that would increase their safety [49]. Indeed, a recent study conducted in Qatar [50] looking at social distancing practices during the COVID-19 pandemic, found that neuroticism was positively correlated with adopting social distancing. Similarly to what was reported above on dietary habits, our study does suggest that the perception of a health risk factor, in those who score high in neuroticism will react in favorable ways towards health. Those who score high in neuroticism will less likely spend their time sitting, in order to avoid risky behavior.

This study has some strengths and limitations. The main strength of the study was that it provided for the first time observational data on associations between diet and PA during the COVID-19 lockdown in Qatar and associations with personality dimensions. The use of an online questionnaire was a quick and cost effective method in gathering data on dietary and PA habits which would not have been possible to do face-to-face due to the lockdown. Both the MEDAS and the IPAQ-SF questionnaires are validated and widely used instruments for assessing MD adherence and PA habits respectively. With regards to limitations, the cross-sectional design of the study does not allow drawing conclusions on cause and effect relationships. Moreover, dietary and PA changes were self-reported and thus may be susceptible to recall bias especially with regards to the 'before lockdown' component. Thirdly, the study population had a high proportion of participants who were single, female students, aged 21-29 years with an undergraduate degree education level, thus limiting the generalizability of the findings. This was due to the oversampling of networks, related to the non-random snowball sampling method and since recruitment was mostly through social media accessed mainly by younger people. Longitudinal studies with representative samples should be conducted to better 
understand the lasting effects of the COVID-19 lockdown and the restrictions put in place on dietary and PA habits, their changes over time and associations with the different personality dimensions.

\section{Conclusions}

The present study provided, for the first time, information on eating habits and PA habits during the COVID-19 lockdown period in Qatar. Our results reveal a reduction in PA and changes in eating habits during the lockdown period. Personality types were associated with dietary habits and PA mainly before rather than during lockdown. Poor dietary habits together with an unhealthy lifestyle can cause serious health problems, therefore adverse lifestyle changes during lockdown periods should be considered carefully. Although our study was conducted in Qatar, our findings may be generalized to other populations with similar demographic characteristics, due to the similarities of lockdown measures across the world. Future work will need to address whether there are long-term changes in diet and exercise habits and how these are associated with different personality dimensions in response to the ongoing COVID-19 pandemic. The findings should be used to develop appropriate personality-tailored lifestyle interventions to reduce unwarranted negative health effects during lockdown periods and generally.

Availability of Supplementary Materials: The following are available online at www.mdpi.com/xxx/s1,

Author Contributions: Conceptualization: T.A., E.P., A.H., and A.P; methodology: T.A., E.P., A.H., and A.P; formal analysis: T.A. and A.H.; investigation: T.A.; resources: T.A; data curation: T.A.; writing-original draft preparation, T.A.; writing-review and editing, T.A., E.P., A.P. and A.H.; supervision, E.P., A.H., A.P. All authors have read and agreed to the published version of the manuscript.

Funding: This research received no external funding.

Institutional Review Board Statement: The study was conducted according to the guidelines of the Declaration of Helsinki, and approved by the Institutional Review Board of QATAR UNIVERSITY (QU-IRB 1081-EA/19, Approval Date: May 8, 2019, renewal until May 6, 2021).

Informed Consent Statement: Written informed consent was obtained from all subjects involved in the study. 
Acknowledgments: The authors would like to extend their gratitude and acknowledgments to all study participants and to Asma Jamil and Rawan Jomat for their technical assistance with the study.

Conflicts of Interest: The authors declare no conflict of interest.

\section{References}

1. WHO Coronavirus Disease (COVID-19) Dashboard. Available online: https://covid19.who.int/ (accessed on 8 February).

2. Singhal, T. A Review of Coronavirus Disease-2019 (COVID-19). Indian J Pediatr. 2020, 87(4), 281-286. DOI: 10.1007/s12098-020-03263-6.

3. Ben Hassen, T.; El Bilali, H.; Allahyari, MS. Impact of COVID-19 on Food Behavior and Consumption in Qatar. Sustainability. 2020, 12(17), 6973. DOI: 10.3390/su12176973.

4. Ministry of Public Health. Controlled lifting of COVID-19 restriction plans. Available online: https:// covid19.moph.gov.qa/EN/Precautions-for-lifting-restrictions/Pages/default.aspx (accessed on December 2020).

5. Górnicka, M.; Drywień, ME.; Zielinska, MA.; Hamułka, J. Dietary and Lifestyle Changes During COVID-19 and the Subsequent Lockdowns among Polish Adults: A Cross-Sectional Online Survey PLifeCOVID-19 Study. Nutrients. 2020, 12(8), 2324. DOI: 10.3390/nu12082324.

6. Zachary, Z.; Brianna, F.; Brianna, L.; Brianna, L.; Garrett, P.; Jade, W.; Alyssa, D.; Mikayla, K. Self-quarantine and weight gain related risk factors during the COVID-19 pandemic. Obes Res Clin Pract. 2020, 14(3), 210-216. DOI: 10.1016/j.orcp.2020.05.004.

7. Martinez-Ferran, M.; De la Guía-Galipienso, F.; Sanchis-Gomar, F.; Pareja-Galeano, H. Metabolic Impacts of Confinement during the COVID-19 Pandemic Due to Modified Diet and Physical Activity Habits. Nutrients. 2020, 12(6), 1549. DOI: 10.3390/nu12061549

8. Mattioli, A.V.; Ballerini Puviani, M. Lifestyle at Time of COVID-19: How Could Quarantine Affect Cardiovascular Risk. Am. J. Lifestyle Med. 2020, 14(3), 240-242. DOI: 10.1177/1559827620918808.

9. Richards, T.J.; Rickard, B. COVID-19 impact on fruit and vegetable markets. Can. J. Agric. Econ. Can. D'agroecon. 2020, 68, 189-194. DOI: 10.1111/cjag.12231.

10. Deschasaux-Tanguy, M.; Druesne-Pecollo, N.; Esseddik, Y.; De Edelenyi, F.S.; Allès, B.; Andreeva, V.A.; Baudry, J.; Charreire, H.; Deschamps, V.; Egnell, M.; Fezeu, L.K. Diet and physical activity during the COVID-19 lockdown period (March-May 2020): results from the French NutriNet-Santé cohort study. Am J Clin Nutr. 2021, Advance online publication. DOI: 10.1093/ajcn/nqaa336.

11. Ammar, A.; Brach, M.; Trabelsi, K.; Chtourou, H.; Boukhris, O.; Masmoudi, L.; Bouaziz, B.; Bentlage, E.; How, D.; Ahmed M.; et al. Effects of COVID-19 Home Confinement on Eating Behaviour and Physical Activity: Results of the ECLB-COVID19 International Online Survey. Nutrients. 2020, 12(6), 1583. DOI: 10.3390/nu12061583.

12. Di Renzo, L.; Gualtieri, P.; Pivari, F.; Soldati, L.; Attinà, A.; Cinelli, G.; Leggeri, C.; Caparello, G.; Barrea, L.; Scerbo, F.; et al. Eating habits and lifestyle changes during COVID-19 lockdown: an Italian survey. J Transl Med. 2020, 18(1), 229. DOI: 10.1186/s12967-020-02399-5.

13. Evers, C.; Dingemans, A.; Junghans, AF.; Boevé, A. Feeling bad or feeling good, does emotion affect your consumption of food? A meta-analysis of the experimental evidence. Neurosci Biobehav Rev. 2018, 92, 195-208. DOI: 10.1016/j.neubiorev.2018.05.028.

14. Scarmozzino, F.; Visioli, F. Covid-19 and the Subsequent Lockdown Modified Dietary Habits of Almost Half the Population in an Italian Sample. Foods. 2020, 9(5), 675. DOI: 10.3390/foods9050675.

15. Al-Mughamis, N.S.; Alayoub, A.A.; Meraj, H.; Waqas, A. Development and validation of Attitude Toward Nutrition Counselling Questionnaire for use among Kuwaiti healthcare professionals. BMC Res. Notes. 2020, 13(1). DOI: 10.1186/s13104-020-4905-9.

16. Huber, B.C.; Steffen, J.; Schlichtiger, J.; Graupe, T.; Deuster, E.; Strouvelle, V.P.; Fischer, M.R.; Massberg, S.; Brunner, S. Alteration of physical activity during COVID-19 pandemic lockdown in young adults. J. Transl. Med. 2020, 18(1), 410. DOI: 10.1186/s12967-020-02591-7.

17. Alomari, M.A.; Khabour, O.F.; Alzoubi K.H. Changes in Physical Activity and Sedentary Behavior Amid Confinement: The BKSQ-COVID-19 Project. Risk Manag Healthe Policy. 2020, 13, 1757-1764. DOI: 10.2147/RMHP.S268320. 
18. Jokela, M.; Hintsanen, M.; Hakulinen, C.; Batty, G.D.; Nabi, H.; Singh-Manoux, A.; Kivimäki, M. Association of personality with the development and persistence of obesity: a meta-analysis based on individual-participant data. Obes. Rev. 2013, 14(4), 315-323. DOI: 10.1111/obr.12007.

19. Mõttus, R.; Realo, A.; Allik, J.; Deary, I.J.; Esko, T.; Metspalu, A. Personality traits and eating habits in a large sample of Estonians. Health psychol. 2012, 31(6), 806-814. DOI: 10.1037/a0027041.

20. Sutin A.R.; Stephan Y.; Luchetti M.; Artese A.; Oshio A.; Terracciano A. The Five-Factor Model of Personality and Physical Inactivity: A Meta-Analysis of 16 Samples. J Res Pers. 2016, 63, 22-28. DOI: 10.1016/j.jrp.2016.05.001.

21. Terracciano, A.; Costa, P.T.Jr. Smoking and the Five-Factor Model of personality. Addiction 2004, 99(4), $472-481$. DOI: $10.1111 /$ j.1360-0443.2004.00687.x.

22. Wilson K.E.; Dishman R. Personality and physical activity: A systematic review and meta-analysis. Pers. Individ. Dif. 2015, 72, 230-242. DOI: 10.1016j.paid.2014.08.023.

23. Al Abdi T.; Andreou E.; Papageorgiou A.; Heraclides A.; Philippou E. Personality, Chrono-nutrition and Cardiometabolic Health: A Narrative Review of the Evidence, Adv. Nut. 2020, 11(5), 1201-1210. DOI: 10.1093/advances/nmaa051.

24. Sutin, A.R.; Luchetti, M.; Aschwanden, D.; Lee, J.H.; Sesker, A.A.; Strickhouser, J.E.; Stephan, Y.; Terracciano, A. Change in five-factor model personality traits during the acute phase of the coronavirus pandemic. PLoS One. 2020, 15(8), DOI: 10.1371/journal.pone.0237056.

25. Specht, J.; Egloff, B.; Schmukle, S.C. Stability and change of personality across the life course: The impact of age and major life events on mean-level and rank-order stability of the big five. J Pers Soc Psychol. 2011, 101, 862-82. DOI: $10.1037 / \mathrm{a} 0024950$.

26. Aschwanden D.; Strickhouser, J.E.; Sesker, A.A., Ji Hyun, L.; Martina, L.; Yannick, S.; Angelina R.S.; Antonio, T. Psychological and behavioural responses to Coronavirus disease 2019: The role of personality. Eur. J. Pers. 2021, 35(1),51-66. DOI: 10.1002/per.2281.

27. Kroencke, L.; Geukes, K.; Utesch, T.; Kuper, N.; Back, M. Neuroticism and Emotional Risk During the COVID-19 Pandemic. J. Res. Pers. 2020, 89, 104038. DOI: 10.31234/osf.io/8c6nh.

28. Zhang, X.; Wang, Y.; Lyu, H.; Zhang, Y.; Liu, Y.; Luo, J. The Influence of COVID-19 on Well-Being. https://doi.org/10.31234/osf.io/znj7h.

29. Cohen, N.; Arieli, T. Field Research in Conflict Environments: Methodological Challenges and Snowball Sampling. J. Peace Res. 2011, 8, 423-435. DOI: 10.1177/0022343311405698.

30. García-Conesa, M.T.; Philippou, E.; Pafilas, C.; Massaro, M.; Quarta, S.; Andrade, V.; Jorge, R.; Chervenkov, M.; Ivanova, T.; Dimitrova, D.; et al. Exploring the Validity of the 14-Item Mediterranean Diet Adherence Screener (MEDAS): A Cross-National Study in Seven European Countries around the Mediterranean Region. Nutrients. 2020, 12(10), 2960. doi.org/10.3390/nu12102960.

31. International Physical Activity Questionnaire. 2002, Retrieved from https://sites.google.com/site/ theipaq/.

32. Hallal, P.C.; Victora, C.G. Reliability and validity of the International Physical Activity Questionnaire (IPAQ). Med.Sci Sports Exerc. 2004, 36(3), 556. DOI: /10.1249/01.mss.0000117161.66394.07.

33. John, O.P.; Donahue, E.M.; Kentle, R.L. The Big Five Inventory-Versions $4 a$ and 54. University of California, Berkeley, Institute of Personality and Social Research, Berkeley, CA (1991).

34. Rammstedt, B.; John, O.P. Measuring personality in one minute or less: a 10-item short version of the Big Five Inventory in English and German. J. Res. Pers. 2007, 41(1), 203-212. DOI: 10.1016/j.jrp.2006.02.001.

35. Veronda, A.C.; Irish, L.A. An examination of eating misalignment: The discrepancy between preferred and actual timing of food intake. Chronobiol.Int. 2020, 0:0, 1-8. DOI: 10.1080/07420528.2019.1692349.

36. Costa, P.T.; Terracciano, A.; McCrae, R.R. Gender differences in personality traits across cultures: robust and surprising findings. J Pers Soc Psychol. 2001, 81(2), 322-331. DOI: 10.1037/0022-3514.81.2.322.

37. Weisberg, Y.J.; Deyoung, C.G.; Hirsh, J.B. Gender Differences in Personality across the Ten Aspects of the Big Five. Front Psychol. 2011, 2,178. DOI: 10.3389/fpsyg.2011.00178.

38. Bagnjuk, J.; König, H.H.; Hajek, A. Personality Traits and Obesity. Int J Environ Res Public Health. 2019, 16(15), 2675. DOI: 10.3390/ijerph16152675.

39. Brummett, B.; Babyak, M.; Williams, R.; Barefoot, J.; Costa, P.; Siegler, I. NEO personality domains and gender predict levels and trends in body mass index over 14 years during midlife. J Res Pers. 2006, 40, 222-36. DOI: 10.1016/j.jrp.2004.12.002.

40. Rodríguez-Pérez, C.; Molina-Montes, E.; Verardo, V.; Artacho, R.; García-Villanova, B.; Guerra-Hernández, E.J.; Ruíz-López , M,D. Changes in Dietary Behaviours during the COVID-19 Outbreak Confinement in the Spanish COVIDiet Study. Nutrients. 2020, 12(6), 1730. DOI: 10.3390/nu12061730. 
41. Reyes-Olavarría, D.; Latorre-Román, P.Á.; Guzmán-Guzmán, I.P.; Jerez-Mayorga, D.; Caamaño-Navarrete, F.; Delgado-Floody, P. Positive and Negative Changes in Food Habits, Physical Activity Patterns, and Weight Status during COVID-19 Confinement: Associated Factors in the Chilean Population. Int J Environ Res Public Health. 2020, 17(15), 5431. DOI: 10.3390/ijerph17155431.

42. Di Renzo, L.; Gualtieri, P.; Pivari, F.; Soldati, L.; Attinà, A.; Cinelli, G.; Leggeri, C.; Caparello, G.; Barrea, L.; Scerbo, F.; et al. Eating habits and lifestyle changes during COVID-19 lockdown: an Italian survey. J. Transl. Med. 2020, 18(1), 229. DOI: 10.1186/s12967-020-02399-5.

43. Giacalone, D.; Frost, M.B.; Rodríguez-Pérez, C. Reported Changes in Dietary Habits During the COVID-19 Lockdown in the Danish Population: The Danish COVIDiet Study. Front. Nutr. 2020, 7, 592112. DOI:10.3389/fnut.2020.592112.

44. Husain, W.; Ashkanani, F. Does COVID-19 change dietary habits and lifestyle behaviours in Kuwait: a community-based cross-sectional study. Environ Health Prev Med. 2020, 25, 61. DOI: 1186/s12199-020-00901-5.

45. Bogg, T.; Roberts, B.W. Conscientiousness and health-related behaviors: a meta-analysis of the leading behavioral contributors to mortality. Psychol Bull. 2004, 130(6), 887-919. DOI: 10.1037/0033-2909.130.6.887.

46. Miguel, F.K.; Machado, G.M.; Pianowski, G.; Carvalho, L.F. Compliance with containment measures to the COVID-19 pandemic over time: Do antisocial traits matter? Pers Individ Dif. 2021, 168, 110346. DOI: 10.1016/j.paid.2020.110346.

47. Lunn, T.E.; Nowson, C.A.; Worsley, A.; Torres, S.J. Does personality affect dietary intake? Nutr. 2014, 30(4), 4039. DOI: 10.1016/j.nut.2013.08.012.

48. Gale, C. R.; Čukić, I.; Batty, G.D.; McIntosh, A.M.; Weiss, A.; Deary, I.J. When Is Higher Neuroticism Protective Against Death? Findings From UK Biobank. Psychol. Sci. 2017, 28(9), 1345-1357. DOI: 10.1177/0956797617709813.

49. Jonason, P.; Sherman, R.A. Personality and the perception of situations: The Big Five and Dark Triad traits. Pers. Individ. Diff. 2020, 163, DOI: 10.1016/j.paid.2020.110081.

50. Abdelrahman, M.K. Personality Traits, Risk Perception, and Protective Behaviors of Arab residents of Qatar during the COVID-19 pandemic. Int. J. Ment. Health Addict. 2020, 1-12. DOI: 10.1007/s11469-020-00352-7. 